\title{
‘Catching ovulation': Exploring women's use of fertility tracking apps as a reproductive technology
}

\begin{abstract}
Smartphones are increasingly entangled with the most intimate areas of everyday life, providing possibilities for the continued expansion of digital self-tracking technologies. Within this context, the development of smartphone applications targeted at female reproductive health are offering novel forms and practices of knowledge production about reproductive bodies and processes. This article presents empirical research from the UK on women's use of fertility tracking applications, known more generally as fertility apps, while trying to conceive. Drawing on material from interviews with women who had experience of using fertility apps, I demonstrate the significance of this particular form of fertility tracking for the embodied shift from pregnancy prevention to actively facilitating pregnancy, participants' sense of self and identity, and how they perceived the reproductive potentiality of their bodies. I argue that fertility apps are significantly involved in making fertility cycles known and thus configuring the pre-pregnant reproductive body.
\end{abstract}

Key words: embodiment, new reproductive technologies, biology, hormones, knowledge, women's bodies 
Journal: Body \& Society

Author: Dr Josie Hamper

This paper focuses on women's use of fertility tracking smartphone applications, or fertility apps, while trying to conceive and explores how this technological practice plays a role in how bodies come to be known as reproductive. Fertility apps provide a digital tool for recording menstruation and bodily signs of ovulation, with the promise of producing more detailed knowledge of the individual's menstrual cycle and predictions of their most fertile days or fertile window in the cycle. While there are various potential uses of self-tracking apps for recording menstruation, including the avoidance of pregnancy, the focus here is specifically on the adoption of fertility apps by women who want to become pregnant. Drawing on interview material from research in the UK, I consider fertility apps as tools of knowledge production and explore how they are involved in making sense of emergent knowledge about the fertile window as a space and time of intervention and opportunity. More broadly, I suggest that understanding women's fertility app use is a productive way to explore the ongoing reconfiguration of reproductive bodies and the complex lived entanglements (Lock and Nguyen, 2010) of technology, bodily knowledge and change, reproductive work, and their relation to shifting senses of self and identity.

This research is underpinned by critical feminist understandings of reproductive bodies as lived through their materialities and bodily form as well as their social construction. Bodies are differently located in space and time, and embodied knowledge is thus a product of particular contexts and knowledge systems that are subject to change (Haraway, 1988). As Judith Butler (1993) has argued, categories of sex and gender are produced through discourse and power as opposed to being natural effects of the body, and there is no natural body that exists independently of or escapes discursive formation. Yet Butler does not deny the existence of material difference and 
Journal: Body \& Society

Author: Dr Josie Hamper

calls for 'a return to the notion of matter, not as a site or surface, but as a process of materialization that stabilizes over time to produce the effect of boundary, fixity, and surface we call matter' (1993:9, original emphasis). The process of materialisation here encompasses the coming together of materiality and discourse in a process of becoming material and gaining the appearance of stability. In her work on hormones, Celia Roberts (2007) has extended these conceptualisations by using the term 'bio-social' to signify the co-construction of the biological and social, or biological and technological, as opposed to the complete implosion of these categories. Critical attention to the materiality of the body forms part of a feminist political endeavour to access a more productive approach to materiality, where materiality is not seen as a constraint but a force that can provoke and incite the social.

The productive tensions between ontological domains is pertinent to discussing reproductive technologies, where humans and non-humans, nature and culture, are enrolled together in an 'ontological choreography' of making babies, parents and other forms of relatedness (Thompson, 2005). Tracing the fragile boundaries between domains that are thought of as ontologically separate affords some insight into how reproduction is imagined and practiced. With the emergence of new reproductive technologies, conception narratives are becoming longer, more complex and more detailed, but still told in ways that make sense according to continuously evolving understandings of what counts as 'natural' in this context (Franklin, 1995). For example, distinctions between assisted and natural conception are complicated in the context of fertility app use, where apps claim to use scientific methods to assist in conceiving naturally. In other words, fertility apps aid natural conception, yet the form of 
naturalness employed by this technique readily encompasses digital fertility monitoring methods that are described as scientific and data-driven.

Fertility apps can be understood as a form of reproductive technology involved, as I explore here, in the continuously evolving intersections between bodies, technologies and reproductive work as these emerge through both normalised and innovative practices of reproduction (Franklin, 1997; Davis-Floyd and Dumit, 1998; Thompson, 2005; Almeling, 2015). While cognisant of the risks attached to framing early efforts to conceive in terms of pre-pregnancy, given that pregnancy is not a guaranteed end-result, participants in this research decisively positioned their fertility tracking in relation to a hoped-for future pregnancy. In this context, fertility apps are a 'hope technology' in that they are emotionally charged with the hope for a future child in an inherently uncertain reproductive future (Franklin, 1997). The future-oriented temporality of fertility apps, where gaining knowledge about fertility is presented as having the potential to improve future chances of pregnancy, is embroiled with medicalised and individualised understandings of risk and responsibility. In an analysis of pre-pregnancy risk discourses in the US, Miranda Waggoner (2015) traced the emergence of a zero trimester of pregnancy where younger women are encouraged to pre-conceive risk and demonstrate pre-maternal responsibility through pre-conception care. Although the US context has different conditions for accessing healthcare than in the UK, the fact that many popular fertility apps are developed in the US suggests some overlap between these geographies of pre-conception health advice within which fertility tracking must be situated.

With advances in digitised self-monitoring practices the landscape of potential data collection is expanding into new spheres of activity, leading to a redefinition of 
Journal: Body \& Society

Author: Dr Josie Hamper

daily living in terms of healthy or unhealthy behaviour (Lupton, 2013; Ruckenstein, 2014; Smith and Vonthethoff, 2016) and impacting on how the body is lived and perceived as measurable and accessible as data (Brown and Webster, 2004). The new modes of visibility and optimisation that are made possible through self-tracking apps emerge from a long history of 'making visible' where seeing has a prioritised position in the production of reliable knowledge. Self-tracking practices, combined with the increased reach of ubiquitous surveillance through smartphones, resonates with Foucauldian analyses of disciplinary power and the production of 'docile bodies' that both internalise the possibility of observation (Lupton, 2012; Rettberg, 2014) and actively participate in the collection and sharing of bodily data (Epstein, 2016). Rachel Sanders' (2016) analysis of digital self-tracking devices explores how these 'technologies of the self' are implicated in promoting public health, fashion and beauty discourses that jointly 'amplify women's sense of obligation to engage in digitally assisted regimes of self-improvement' (2016:51). Crucially, these digital regimens of discipline and normalisation intensify opportunities for individuals to actively position themselves, and continuously measure their level of 'improvement', against normative ideals of health and femininity that have patriarchal underpinnings.

Building on socio-material perspectives on the entanglements of human bodies and technology, and feminist cultural theories of female embodiment, Deborah Lupton (2014a, 2015) has considered how fertility apps play a particular role in constructing digitised reproductive citizens who are highly aware of and attentive to their reproductive health. Fertility apps follow the rhetoric of 'enabling' women to gain better knowledge about the reproductive body through data science, thus implying that the knowledge women gain from experiencing and observing their own bodies in other 
Journal: Body \& Society

Author: Dr Josie Hamper

ways is lacking or incomplete (Lupton, 2014a). Digital fertility tracking relies on the idea that 'better data' offers a means for gaining control over reproductive health and lives. Yet recent critiques of the accuracy claims made by fertility apps call into question the reliability of these devices, particularly when used as a contraceptive method, by stressing the significant divergence in their effectiveness between perfect-use and typical-use (ASA, 2018). ${ }^{1}$ References to perfect use in this context are attached to highly value-laden and individualised notions of (ir)responsibility, where some women and their bodies and lifestyles are positioned more favourably in their ability to sustain the required intensive and daily labour of perfect fertility monitoring. Fertility apps evoke certain modes of feminine embodiment that configure perfection as dependent on dominant codes of strict bodily discipline and conduct, and they can therefore be seen as part of a wider apparatus of meaning and calculation that brings the idea of 'the perfect' to life as a benchmark for comparison between women (McRobbie, 2015). This paper emerges from a broader research study that explored how fertility and pregnancy apps are involved in the making of reproductive bodies, babies, mothers and relations, for largely white middle-class women in London. Here I focus on how fertility apps are significantly involved in configuring continuously changing modes of pre-pregnant embodiment, including the lived dynamics of how fertility tracking in an app becomes meaningful reproductive work in a culture that values self-expertise and control. I draw on 27 anonymised interviews with 15 women aged 30 to 44, all of whom were in long-term heterosexual relationships and had experience of using fertility apps with the intention to conceive. Two participants had used an app as a precursor to fertility treatment. Participants were invited to take part in two interviews, although some preferred one longer interview over meeting twice, and interviews took place 
Journal: Body \& Society

Author: Dr Josie Hamper

either in the participant's home or a café. Participant recruitment methods included the distribution of flyers in cafés, community spaces and online groups, as well as in-person recruitment at parent groups in east and north London. These locations were attended by a diverse set of people, and participation in the research was open to women of any ethnic, religious and socioeconomic background, yet those who volunteered to take part formed a participant group that was largely white and broadly middle-class. ${ }^{2}$

This pattern of participation shaped the study's focus on the specificities of fertility tracking for white middle-class women's reproductive experience. In discussing their app use with me these women demonstrated a strong sense of empowerment in their reproductive decision-making, in terms of their capacity to choose not to have children at certain life stages (through contraception or pregnancy termination), their access to healthcare and fertility treatment when needed, and the importance they assigned to pregnancy planning. The importance of planning for the future is particularly pertinent when considering fertility tracking with the intention to conceive. Planning pregnancy assumes an active choice in determining the 'right time' to have a child (Martin, 2017), which is tied to a neoliberal idea that women are singularly responsible for anticipating and managing family life alongside a fulfilling career (Rottenberg, 2014). Reproductive choice and entitlement are, however, deeply stratified and embedded in structural constraints that shape the meanings and values assigned to reproductive futures (Ginsburg and Rapp, 1995). Racial and economic privilege position white middle-class heterosexual couples as the primary intended consumers of fertility technologies (Davis, 2009) and determine whose reproductive capacity is intervened in most excessively (Bridges, 2011). The 'dispositif' of self-regulation that app users are recruited into 'envelopes' women differently along intersections of citizenship, class, 
Journal: Body \& Society

Author: Dr Josie Hamper

race, age, family formation, sexuality, body size and other identity markers (McRobbie, 2015), shaping women's access to and encounters with monitoring technologies, including smartphones, in ways that potentially reproduce existing health inequalities (Lupton, 2012). The fact that the participants in this study were actively trying to conceive within long-term heterosexual relationships aligns them with the idealised heteronormative image of feminine reproductive citizens who are able and willing to engage in their app's prescribed practices of fertility monitoring (Lupton, 2015; Wilkinson et al., 2015).

In this paper I explore how participants negotiated the objectification of their bodies in a neoliberal sociopolitical context where choice and the imperative to be in control of one's fertility is felt as both a freedom and constraint (Rottenberg, 2014). In order to address this, I firstly outline the nature of fertility apps, including how they work and what they claim to do, and I consider how participants differentiated apps from other non-digital tracking methods. I then explore how apps were entangled with women's shifting modes of embodiment and how their practices of fertility tracking were underpinned by individualised rationales that emphasise the connections between knowledge, empowerment and control.

\section{Fertility apps in practice}

The practice of self-monitoring fertility signs is longstanding and established in some women's health communities (DeNora, 1996), yet the broad uptake of fertility apps indicates that they are involved in expanding the reach of these methods to a wider population. Fertility apps draw broadly on the principles of fertility awareness methods, 
Journal: Body \& Society

Author: Dr Josie Hamper

which involve tracking bodily symptoms to identify the days prior to and immediately following ovulation. The more advanced apps combine calendar-based methods of calculating ovulation with the observation and recording of a variety of physiological symptoms that respond to cyclical fluctuations in oestrogen and progesterone levels. In addition to the dates of menstruation, sympto-thermal monitoring methods require the daily recording of basal body temperature as well as changes in cervical mucus. Other apps include options to monitor some or all of the following: sexual intercourse, cervix dilation, ovulation and pregnancy test results, medications taken, fertility treatment protocols, breast tenderness, mood and energy levels. While extensive data-entry options reflect a wider range of possible fertility symptoms, there is also an underlying assumption that all these symptoms are connected to hormonal fluctuations, thus aligning with critical perspectives on the huge amount of 'explanatory work' that hormones do in all aspects of women's lives (Roberts, 2007:191).

Once a certain number of menstrual cycles have been recorded, the app identifies a pattern upon which it bases future predictions of menstruation and ovulation dates. In the context of assisting conception, these predictions are used to plan sex or insemination on the app user's most fertile days. ${ }^{3}$ Facilitated by the highquality touch-screens characteristic of contemporary smartphone devices, fertility apps consistently involve some kind of data visualisation, either in the form of calendars where data is layered onto the relevant date or charts where the y-axis represents temperature and the $\mathrm{x}$-axis the day of the month. Diagrams, calendars and charts are culturally familiar ways of isolating and reading bodily processes (Sawchuk, 2000; van Dijck, 2005) and the temporal arrangement of these is especially relevant in the context of reproduction where timing is central to notions of planning and control (Simonds, 
2002; Downe and Dykes, 2009). The purpose of these charts is to create a perception of order over an individual's menstrual cycle - to make it transparent - and ultimately render this bodily process as fully knowable and subject to action.

Whereas the marketing material for fertility apps often emphasises the power of fertility data, prediction algorithms and smartphone computing, participants more frequently talked about the novelty of apps in terms of how readily they fit into everyday routines. Apps assist in normalising fertility tracking by presenting it as a regular and unobtrusive part of everyday life, and participants' descriptions of the work of fertility tracking drew on notions of ease, efficiency and personalisation. Drawing on Ruth Schwartz Cowan's (1983) conceptualisation of the 'work process', tracking fertility signs in an app may be considered as one part in a broader and increasingly complex set of related tasks; from keeping a smartphone's battery charged to interpreting the data and seeking advice about ambiguous results.

\section{Convenience and the work of fertility tracking}

Participants conceptualised fertility apps as related to but also tangibly different from manual fertility tracking with a 'pen and paper'. Such comparisons present an account of technological change where apps extend established fertility tracking methods into new spatial and temporal routines. Tracking fertility in an app made sense to participants in a world where the technological affordances of smartphones are seen as perfectly suited to contemporary lifestyles, mobility and the organisation of everyday life (Kitchin and Dodge, 2011). Linked to this was the appeal of seamless access to information from 'anywhere' and a sense of stability to the digital archive of fertility 
Journal: Body \& Society

Author: Dr Josie Hamper

data. The idea that manual fertility tracking would require an additional level of organisation was widespread among participants and reiterates messages in the advertising of apps, where paper-based fertility tracking is portrayed as arduous work, thereby presenting a 'problem' that apps can provide a 'solution' to.

Comments that connected phones and life were frequent in interviews. One woman explained that her fertility app helped her gain 'a sense of getting a bit of control over my life' and another talked about her fertility tracking as 'housekeeping', thus clearly connecting this to the broader organisation of domestic activities and suggesting how the tasks that make up domestic work change alongside changing technology (Cowan, 1983). A third participant stated that apps are different to other online information sources because '[smartphones are] generally sort of an extension of yourself a bit more, so there's that... there's that opportunity there for it to be very personalised and private.' Constant proximity, personalisation, convenience and ease of use were central to the appeal of fertility apps. Crucially, the subtle incorporation of apps into people's daily routines offers app companies new access to vast amounts of detailed data on bodies and lives (Lupton, 2013, 2014a). Apps are connected nodes in a wider digital network, which has implications for intimate reproductive work that takes place at the scale of the body, where the translation of bodily processes into quantitative data reconstitutes these as valuable in the context of a digital data economy (Lupton, 2015). While some participants acknowledged how the sharing of personal data with apps and associated companies might compromise personal privacy, the dominant narrative was that fertility apps were private due to them being on personal phones. 
Journal: Body \& Society

Author: Dr Josie Hamper

Some participants expressed a sense of reassurance in that their app would remind them to enter data if they forgot to do so and a sense of relief in handing over the calculative work of fertility monitoring to technology (Wilkinson et al., 2015). The acts of taking temperature measurements and observing physiological fertility symptoms are the same with or without an app, but having an app made the recording, analysis and interpretation of data easier. The emphasis on apps as an easy and convenient technology is important to justifications for fertility app use, yet it presents a paradox in that the majority of participants would not have undertaken such extensive tracking if it were not for having an app. Lucy emphasised the ease of using a fertility app versus manual fertility monitoring:

Otherwise I would have been noting it [temperature measurements] down on a piece of paper and doing my own little graphs. Because I quite like, I wanted something very visual. I'm quite a visual person. And otherwise I'd have done it myself, and I wouldn't have done it, you know, if I was going to write it all down on a piece of paper. Whereas you roll out of bed in the morning, take your temperature and jot it down. It's so much easier than trying to make a note of something.

Following a longstanding trend of positioning new technologies in terms of their efficiency (Cowan, 1983), Lucy's statement that she would not have engaged in manual fertility tracking without an app demonstrates how fertility apps create extra labour for women while being talked about as a labour-saving technology.

\section{Personalised advice}


The 'pushiness' of apps, which refers to their use of push notifications and other means of gaining and retaining users' attention, can introduce new spatial and temporal modes of self-care (Lupton, 2017). In addition to fertility signs, some apps ask the user to input details about broader health and lifestyle factors, such as exercise levels and diet. This information does not form part of the fertility prediction or analysis but it can influence the personalised advice that is offered via the app, which in turn provokes selfreflection in the app user. Shannon explained that she was surprised at how much information her app 'wanted' from her and she was not convinced that all of this information was relevant to fertility. Furthermore, she felt that the advice offered was shrouded in moral judgement:

The other night I went to a wedding and yes, I did drink alcohol, and it [the app] is like 'oooh limit your alcohol' and I was like 'well I was at a wedding!' [Laughs] 'give me a rest!' kind of thing. So yeah, I think it probably triggers their hints and tips, which as I said are quite patronizing. Like 'did you exercise?' - 'No' - 'Well you should' - 'Yes, I know I should but I didn't.'

Shannon highlighted the annoying and potentially problematic nature of these kinds of behavioural recommendations. The way in which she imagined this interaction with her app as a conversation shows how apps are seen as having the ability to speak back at the user in a way that is responsive and personalised. She described how the app sometimes made her feel conscious about whether she was doing the 'right' things to get pregnant, or conversely that she was not doing enough. 
Journal: Body \& Society

Author: Dr Josie Hamper

The advice offered by fertility apps may be personalised at one level, but it also emerges from aggregated data that forms the basis for generalised advice and knowledge claims about reproductive bodies that are fed back to the app user. Shannon questioned the all-encompassing nature of her app and challenged the connections that were being drawn between changes in mood as always related to hormonal fluctuations:

I don't enter most of the information, but you know, it's things like your mood, your... I don't know, any physical symptoms. And I think that, I feel that you can read too much into that. Yeah, I'm feeling a bit shitty because something is a bit shitty. It's not all about fertility. I think, yeah I can see it's... So I don't use it much because it's got all this stuff in it. 'Low mood? Could that be this?' I find it a bit much really.

There is an assumption that fertility awareness should initiate action in all areas of life. Yet participants generally saw themselves as being in a position of authority in choosing what advice to take or ignore from their apps or opting to withhold certain pieces of information that they felt were too personal or irrelevant. This sense of choice indicates the complexity of agency, where apps can have a significant impact on people's thinking (van Dijck, 2009) while also being open to interpretation and the 'social tactics' of app users (Gillespie, 2014:183). Several women, like Shannon, explained how they had chosen to ignore advice that they felt was overly prescriptive, moralising or negative. Although no technology is passive, apps are particularly 'vocal' in their ability to offer personalised reminders in a timely and unprompted manner, and in this way, they 
Journal: Body \& Society

Author: Dr Josie Hamper

intervene in and engender particular self-governing identities (Lupton, 2013; Sanders, 2016).

\section{Shifting modes of embodiment}

In her ethnography of women preparing for fertility treatment, Charis Thompson (2005:192) developed the concept of 'anticipatory socionaturalisation' to describe the temporal dimension of accumulating knowledge and enacting a certain subject position prior to becoming a fertility patient. During this process prospective patients' thinking about and awareness of fertility changes dramatically; in particular Thompson highlighted how these individuals will have developed an increased awareness and knowledge of their menstrual cycle, evaluated their own unique likelihoods of conception and hypothesised about why they are experiencing difficulties conceiving. Through a process of 'unblack-boxing' the body, women find new ways of accessing body parts and processes and making these relevant to their conception efforts.

For the women involved in this research, fertility apps were tied up with a commitment to learning about their own bodies in preparation for a hoped-for pregnancy. Fertility apps played a significant role in how participants gained general knowledge of reproduction as well as knowledge pertaining to their individual fertility cycle. For the app user, this may be thought of in the following terms; this is what menstrual cycles are like and this is what my menstrual cycle is like, where apps are involved in forging connections between a general understanding of menstrual cycles and the individual body. This dual process of knowledge production was central to participants' experiences of fertility tracking as well as their shifting modes of pre- 
Journal: Body \& Society

Author: Dr Josie Hamper

pregnant embodiment. In the remainder of this paper I explore the implications of fertility tracking in an app for participants' sense of self and how they understood the reproductive potentiality of their bodies.

\section{Relearning biology}

Many participants described feelings of uncertainty and confusion during the transition from preventing pregnancy to trying to achieve pregnancy, and the notion that reproductive biology is learnt at school and later forgotten came across in several interviews. The school emerged in these accounts as an important site in constituting the female reproductive body in highly universalising terms, including how to construct a healthy future correctly with a particular focus on teenage pregnancy prevention (Longhurst, 2008). Tessa, who had used a fertility app prior to two pregnancies, explained that her app offered 'a kind of biology lesson' like the ones she received at school:

I think when I learned female biology aged 16 or 17 in school, I mean although you have periods by then you don't think about pregnancy and all that stuff, so you remember it in an exam sense but then you kind of forget quite quickly. You know how not to get pregnant and so it doesn't really matter... So it [the app] was a bit of a refresher course. There definitely was information on there. I remember reading how eggs come out and whatever, that kind of stuff, how that stuff works. But I didn't learn it for the first time, I think I just learned it in a different context. 
Journal: Body \& Society

Author: Dr Josie Hamper

A contrast is presented in Tessa's account between knowledge gained in a school context or, in her words, knowledge that is learnt in 'an exam sense', and knowledge that is useful in her current situation. ${ }^{4}$ Importantly, she emphasised that much of the knowledge gained through her fertility apps was not new but that it was being applied in a new way; that is, to her own, contextual, lived body and life situation.

There is a sense that learning reproductive biology in textbook form is isolated from the lived experience of reproduction (Martin, 1987, 1991), which echoes analyses of how pregnancy texts decontextualise knowledge about fertility and pregnancy from women's prior histories and knowledge about the body (Marshall and Woollett, 2000) and how medical textbooks represent reproduction as purely biological (Roberts, 2007). For Vera, who used a fertility app prior to fertility treatment, relearning about fertility involved gaining an understanding of the realities of and potential difficulties involved in trying to conceive. She reiterated the school as a site of biology education:

Like I had a really vague understanding from biology at school but you know, umm... I think because when you're in your teens it's drummed into you that you can get pregnant at any time! [Laughs] 'Don’t have sex!' So yeah when your focus starts to shift the other way you sort of find out a bit more about how that isn't really the case.

Vera's account positions her changing understanding of reproduction in relation to the perceived continuously fertile teenage body. Vera noted a 'shift the other way' in relation to her thinking about the likelihood of pregnancy, which highlights shifting subjectivities across the life course (Almeling, 2015) and the extended temporality of coming to know the body differently at different stages of life (Teather, 1999). Referring 
Journal: Body \& Society

Author: Dr Josie Hamper

to teenage pregnancy in this context indicates how pre-pregnant embodiment emerged in relation to other bodies, including the fertile body of the teenager that can get pregnant 'at any time' and the future body that is marked by fertility decline (Martin, 2017). In this way, bodies are products of relations between other bodies that are differently located in space and time, and complexly embodied in the present.

The shift in understanding the reproductive body was experienced differently across the participant group depending in part on each individual's contraceptive history, which was often complex and involved a range of contraceptive methods at different points in time. For women who had been on hormonal contraceptives the decision to try to conceive involved a significant reconfiguration of knowledge about fertility as well as physical bodily change through the gradual return of an unregulated menstrual cycle. In particular, participants who had been taking the contraceptive pill for many years described having a cycle of withdrawal bleeds during monthly pill-free intervals, usually referred to as 'periods' for simplicity, which followed a highly controlled and predictable timeframe.

For others, hormonal contraceptives had stopped them bleeding altogether, which is a common side effect of such methods. Shannon explained how the absence of periods while on the pill had contributed to a loss of knowledge about fertility and prompted a process of educating herself through an app and the internet:

Yeah well for a long time I've been on a pill called Cerazette, which stopped me getting periods, so I had no idea. And I'd been on that for quite a few years, like five years or something [...] Obviously we were thinking about trying for a baby, and I had no idea to be honest how menstrual cycles worked. I couldn't like, you know, you learn it at school 
Journal: Body \& Society

Author: Dr Josie Hamper

and then you forget, and I had absolutely no idea when my fertile period was. So I think I looked up, you know, I was Googling that and then I thought 'okay this app thing would be a good idea.' Yeah, so I downloaded one to track.

Kate, who had used both the coil and contraceptive pill for periods of time, reflected on whether being on the pill had impacted on her experience of her own body and fertility more generally:

[The pill] stopped my periods, which is quite interesting and apparently quite common. But again, I just wonder looking back if that was a bit of a removal from... you know, experiencing periods, or experiencing... or just having that kind of reminder that this process was going on in my body.

Kate talked about her periods as a reminder of the reproductive capacities of her body, and how the amount of time that had passed without experiencing periods had resulted in her feeling removed from this bodily process. Following this, she described a clear sense that when the decision was made to try to conceive she lacked basic knowledge about her own fertility cycle and she started using an app to gain an understanding of the timings of ovulation:

[The app] was a way for me to start tracking because I read, I read online about, I mean I was completely, amazingly ignorant about when I would be fertile, and I used that to help when we were trying. 
Journal: Body \& Society

Author: Dr Josie Hamper

Similarly, Lucy described her fertility tracking against a background of being on the contraceptive pill for over ten years:

Mmm, it was really hard because I was so out of touch with my body. That sounds really hippy-ish but I'd, from the age of 15 I'd been on the pill, and I had a very sort of simple... I knew when it was going to be, I knew when my period was going to be, I wasn't bothered about things, I never thought about things like ovulation because I didn't need to. So one of the reasons I used it [the app] was because I felt completely out of touch with my body.

The emphasis in these narratives on lack of knowledge about fertility is important because not only was this offered as a justification for why apps were needed in the first place, but it also says something about how women perceived success in conceiving as dependent on acquired knowledge and skills. Whereas previously the main reproductive concern of participants had been how not to get pregnant, fertility apps were embedded in a shift towards actively facilitating pregnancy through reconnecting with the reproductive potentiality of the body. Particularly for those who had adopted hormonal contraceptive methods, tracking the gradual return of menstruation offered a means of identifying the physical return of a 'natural' or 'normal' menstrual cycle, which in turn was entangled with the reframing of ovulation as significant in efforts to conceive.

\section{Itinerising the body}


A focus on gaining clarity and awareness was central to many conversations about participants' motivations to track their fertility, which was connected to a process of 'uncovering' fertility cycles and patterns that were not previously known. Alongside experiences of re-contextualising fertility knowledge, women also spoke about a process of constructing disembodied, objective knowledge of reproductive processes. For instance, Kelly explained how her fertility app helped her feel confident that she was giving herself the best chances to conceive: 'The Period Tracker one, I just think it really helped me sort of, get clarity. Just to know the science of it, rather than literally stabbing in the dark.' Kelly's interest in the 'science' of conception is significant and signals the appeal of scientific knowledge and expertise that characterises many contemporary parenting cultures (Lee, 2014). More generally, appealing to science characterises a distinguishing element of the biological citizen who plays an active role in learning about and harnessing biological processes for future-oriented objectives (Rose and Novas, 2005).

Objectifying or translating biological processes into data allows these to take on new meaning as they are brought into new sites or used strategically towards a particular purpose. Configuring the menstrual cycle and ovulation as a bio-social actor offers a means of understanding how these processes are active and significant in producing the biological possibilities of conception, while also appreciating how these 'co-act' (Roberts, 2007) with lived experience, social understandings of reproduction, and complex choreographies of humans, timings and technologies. Kaela noted how her commitment to tracking over a long period of time enabled the app to make relatively accurate predictions of her fertile window. She combined these predictions with ovulation tests, explaining: 'That way I know I'm eventually going to catch my ovulation 
Journal: Body \& Society

Author: Dr Josie Hamper

when it happens.' In her accounts of fertility monitoring, Kaela objectified 'my ovulation' as something that she must 'catch' and make knowable and thus she positioned herself as active in how she used technologies to understand and work with her body. Thinking through the complexities of the lived body can disrupt distinctions between activity and passivity, where ovulation can both act and be acted on.

In addition to learning how reproductive processes work, participants talked about acquiring new language and terms to describe reproductive processes and the body, including ovulation, the luteal phase, cervical mucus, core or basal body temperature, eggs, luteinising hormone, fallopian tubes, follicles, ovaries and ovarian cysts, and much more. Sometimes, the knowledge produced was significant but partial, as is evident from the following exchange with Shannon:

Shannon: I guess that [the app] forced me to do a bit of research around... well... I can't remember what it was called, but the phase, I don't know... You probably know better than me.

Interviewer: The, I never quite know how to pronounce it, the... luteal phase?

Shannon: Yes, that was the one. I didn't even know it was a thing. It made me feel a bit more knowledgeable and in control of that?

The luteal phase (the fertile period between ovulation and next menstruation) has not suddenly become more real than it was all along, but it has gained new relevance in that it has a function and can be acted upon in Shannon's attempts to conceive through the timing of sex during this period. This echoes the ways in which fertility patients come to 
Journal: Body \& Society

Author: Dr Josie Hamper

'itinerise' their bodies in new terms (Thompson, 2005). Yet it is noteworthy that the mutual hesitancy in verbalising the term 'luteal phase' signals a certain awkwardness around unfamiliar language in fertility apps, and indicates how 'the phase' created by the app does not necessarily translate easily into other contexts or conversations, such as the interview. Downloading an app at these very initial stages of trying to conceive was the first part of a process whereby participants' most fertile days were made to appear and produced as 'functional stages' (Thompson, 2005:194) of significance in their efforts to conceive (also Han, 2013). This facilitated new access to body parts and processes that are relevant to conception, as well as how to think about the temporality and connectedness of these in terms of cycles and phases. Participants' interactions with fertility apps were important in assigning the reproductive body new meaning and relevance, thus producing 'new' bodily processes that were previously less (or differently) known.

The visual element of tracking was central to participants' perceptions of patterns in fertility data and many described how their fertility tracking had uncovered symptoms and patterns that they were not previously aware of. Lucy, who only used the fertility app for a short amount of time given that she conceived very quickly, described how she could gradually see that her periods followed a pattern:

I did end up finding the charts quite interesting. And one thing I was able to see over time, because I thought I had very irregular periods, and actually over time I was able to see they were about every six weeks. It just felt irregular to me, but I could see it was starting to fall into a bit of a pattern. 
Journal: Body \& Society

Author: Dr Josie Hamper

This evidence of a pattern influenced how Lucy described her menstrual cycle as no longer being irregular. Crucially, the pattern presented by the app offered a more accurate, algorithmically sanctioned knowledge that effectively replaced Lucy's own self-knowledge of having an irregular menstrual cycle. Through its visualisation the menstrual cycle becomes external to the body and something that can be seen. The way Lucy talked about her periods 'falling into' a pattern gives a sense of how this pattern is constructed jointly by her and the app; rather than uncovering a pattern, the pattern started to fall into place when the tracking and analysis methods worked together over time. In this way, ovulation is constituted through a particular technoscientific visual culture of tracking and quantification; while the pattern produced through fertility tracking remains seen as a natural cycle, it is also a disciplined cycle that has been measured and traced through information (Brown and Webster, 2004).

Making use of biological processes refashions these as tools that can be used towards enhancing chances of reproductive success and thus complicates the distinction between biology and technology (Roberts, 2007), where the menstrual cycle becomes something that can be used towards certain means. For Lucy, gaining knowledge about her body was connected to gaining a sense of control in an out-ofcontrol situation:

[Fertility tracking] was a way of me exercising a bit of control over a situation I felt was slightly out of my control, and also trying to work out what was going on with my body so that I could make the most use of my body. 
Journal: Body \& Society

Author: Dr Josie Hamper

The idea that by knowing her body Lucy could 'make the most use' of it demonstrates that a shift in thinking about the reproductive body has taken place where the menstrual cycle has been brought into a new system of intelligibility. Preoccupations with improving one's chances through self-monitoring and education are connected to a future-oriented biopolitics whereby the future is continuously reconstituted in the present moment (Evans, 2010), and where pre-emptive actions now may pay off in the desired reproductive future (Waggoner, 2015). Gaining information and bodily expertise through self-tracking is presented as beneficial and key to optimising one's own life (Sanders, 2016), hence the adoption of self-tracking devices speaks to a strong imperative to engage with reproduction in a highly individualised, technological and immediate way (Lupton, 2013; Smith and Vonthethoff, 2016). This kind of digital selfsurveillance is normalised and persuasive in its references to discursive regimes of future risk management, control over personal goals and reproductive responsibility (Lupton, 2014b).

\section{Disorderly cycles}

Participants for whom the app had 'worked' described how they felt reassured when they could identify a cycle that followed a regular pattern. Several expressed great relief from having textbook fertility, which not only confirmed that their fertility cycle was working as it 'should' but also that their method of fertility tracking was working as they intended. Participants had a clear image of what their fertility data 'should' look like, where menstruation and other fertility symptoms were imagined as displaying a continuous cycle over time with defined peaks in temperature at even intervals. There 
Journal: Body \& Society

Author: Dr Josie Hamper

is a distinct sense of bodily rhythm to graphic representations of fertility data, where the cyclical peaks in basal body temperature signify 'normality' and each identified ovulation is presented as a significant moment in a broader project of trying to conceive. Participants were well rehearsed in comparing their fertility data to a standard fertility cycle, indicating how fertility tracking has particular ways of making visible normative bodily processes and bringing these into contact with individual selfmonitoring practices (DeNora, 1996). In this context, healthy fertility is aligned with normative ideals of feminine embodiment such as those pertaining to 'healthy' weight, diet and exercise (Sanders, 2016), indicating how menstrual cycles and hormones are involved in social and political articulations of femininity and difference relating to women's reproductive lives (Roberts, 2007).

Some participants were not able to identify patterns in their fertility data and thus had a heightened awareness of themselves as not being textbook. For these participants, their experience of fertility apps involved a complex and tenuous process of knowledge construction where the objective of increased clarity was mired with feelings of confusion and failure. The discussion of Shannon's experience earlier in this paper highlighted how her fertility tracking practices were about educating herself about conception and her body; however, once Shannon returned to fertility tracking following a miscarriage and the birth of her first child, she found that a disjuncture emerged between different forms of knowledge that did not align:

I'm in this situation, so I started using Glow again about two months ago. Shortly after we spoke actually. And it predicted my first cycle correctly and basically, I'm in a situation where my period is four days late according to the app, but I've done a 
Journal: Body \& Society

Author: Dr Josie Hamper

pregnancy test and it says I'm not pregnant, so now I'm like 'aww I'm confused by this app again.' I don't totally trust it but I don't know if that's because I'm not as regular as I thought... or... I don't know...

Confusion sometimes came about when apps gave unexpected results or predictions that did not match women's self-knowledge and there was a tendency for participants to place the responsibility for incoherence on themselves, saying that it must be due to their irregular cycles or deliberating whether their data input was extensive and accurate enough.

Vera undertook periods of detailed fertility monitoring, including daily temperature measurements, over the several years that she tried to conceive. Rather than leading to greater clarity the app further complicated an already ambiguous and uncertain situation, and ovulation remained an elusive event. Initially Vera thought that tracking her fertility would be a positive experience:

I thought that it [fertility tracking] would sort of... give me some control I suppose... Umm... Just so, I suppose so I could feel like I was doing the right things.

In extension to this she explained that she liked knowing that she was being proactive and doing, as she said, something 'positive to try to get pregnant' rather than 'just not preventing it.' The temperature charts in Vera's app were not as the model charts she had seen in fertility awareness educational material and she could rarely identify any clearly defined peaks in her temperature measurements. She described her charts as 'uppy downy' and went on to explain: 
I think if I had been a textbook case I would have found it [the app] a bit more useful, but I wasn't so it just felt a bit like... And then at one point I did the ovulation kit type things [...] and they were also, they basically said I didn't ovulate at all, which wasn't true. So I was like 'oh well' they're not helpful either so I'm obviously just a bit odd in some way and the easy, straightforward ways of monitoring aren't going to work for me, so I might as well not bother, kind of thing.

Although fertility apps claim to pick up on unique cycles, including those that are irregular, apps are to an extent a one-size-fits-all technology in that they configure a particular user with a body that presents the 'right' symptoms (Oudshoorn, cited in Clarke, 2000). The fact that the combined fertility tracking and ovulation tests did not identify a clear cycle meant that there was no fertile window for Vera to act on. She interpreted this as meaning she was odd, which in turn spoiled the sense of empowerment and control that she had hoped to achieve.

More detailed knowledge and a heightened awareness of fertile windows also meant that there was a heightened awareness of the number of conception opportunities that had been missed and the length of time spent actively waiting to conceive. Kate, who had tried to conceive for over two years before becoming pregnant, described this experience of extended waiting:

...when we started trying I sort of became very aware of it [long cycles] very quickly because I was kind of waiting for this opportunity [ovulation], which wasn't coming as often, or as regularly as I wanted it to. Sometimes it would go... I do remember, although I'm not sure I'm remembering... I've got it on my app still I guess... if I'm remembering 
Journal: Body \& Society

Author: Dr Josie Hamper

the right cycles, but sometimes it would be about 50 days. So nearly double, I guess, of what would be normal. Yeah, and that was, that was frustrating, that they were so long.

Whereas fertility apps are quick to explain and claim a part in successful conception by, for instance, including pregnancies in an app's success rates, they are limited in their ability to explain situations where conception does not happen. Kate recounted the emotional strain that she experienced while trying to conceive and how, over time, her app became visual evidence of the absence of pregnancy. Embodied experiences of fertility tracking were characterised by obstacles and uncertainties, which stands in contrast to the idealised clarity, awareness and self-expertise that is emphasised both in participants' descriptions of fertility apps as well as the advertising materials for these tools.

\section{Conclusion: Fertile windows}

In this paper I have explored the role of fertility apps in how the reproductive potentiality of participants' bodies did or did not come into being through the emergence, or absence, of fertile windows in the menstrual cycle. These windows of opportunity did not simply appear in an app, rather they required sustained work over time in order to build an archive of past fertility-related data upon which future fertile days can be algorithmically predicted. The notion of a window offers a metaphorical device for conceptualising fertility tracking; apps can be thought of as windows onto internal body parts or processes that would not otherwise be visible in the same way; the identification of fertile days constitutes a fertile window in the menstrual cycle; and 
Journal: Body \& Society

Author: Dr Josie Hamper

trying to conceive presents a window in the reproductive life course where women's bodies and behaviour are subjected to particular forms of intervention. Attempting to gain a sense of control over an uncertain bodily process underpinned many of the participants' experiences of fertility tracking, indicating how apps work through the encompassing of women within feminised regimens of self-observation and calculation (Sanders, 2016). Attached to this desire for control was a cultural imperative for action, where the emergence of a fertile window would prompt a spatio-temporal arrangement of acts, involving the timing of sex, ovulation tests or health-promoting behaviour, in order to 'catch' ovulation when it happened. Extending out from an intense awareness of the current state of fertility, the daily labour of fertility tracking takes place within a broader discursive context that emphasises reproductive time pressure relating to age (Martin, 2017) and places women as individually responsible for their reproductive futures (Lupton, 2014b; Waggoner, 2014).

Trying to conceive is thus understood as both a biological and social process that connects reproductive experience and knowledge across extended space and time (Almeling, 2015). Participants described a highly demanding cultural context of fertility tracking involving overlapping attempts to relearn biology, itinerise the body in relevant terms and make sense of divergences from the standard fertility cycle. Tracing these practices demonstrates how fertility apps intersect and intervene with numerous other self-monitoring technologies (such as ovulation tests) and 'newly appeared' body parts and processes (such as the luteal phase), across multiple temporal scales (from contraceptive histories to bodily cycles and imagined reproductive futures) and spaces (including the school, home and clinic). These dimensions of trying to conceive often did not come together neatly in participants' narratives but rather involved a great deal of 
Journal: Body \& Society

Author: Dr Josie Hamper

effort to make sense of tensions in the knowledge produced. While apps are situated in a longer history of fertility monitoring practices and technologies, the personalised and responsive nature of apps is significant in engendering new possibilities, such as those afforded by their connectedness to data storage and sharing processes combined with their everydayness and how apps are readily embedded in routine. Fertility apps, as I have shown, have the potential to subtly intensify certain aspects of embodied reproductive experience, awareness and knowledge, and change the ways in which women relate to and know their bodies through data. Engaging with their use is thus critical to understanding the intricate lived experiences of everyday reproductive technologies and the complex intersections between bodies, technologies and reproductive work.

\section{Acknowledgements}

I am very grateful to the journal editors, four anonymous reviewers and Catherine Nash for their insightful comments on this paper. This work was supported by the Economic and Social Research Council [grant number ES/J500124/1]. 
Journal: Body \& Society

Author: Dr Josie Hamper

\section{References}

Almeling, Rene (2015) Reproduction. Annual Review of Sociology, 41: 423-442.

ASA (Advertising Standards Agency) (2018) ASA Ruling on NaturalCycles Nordic AB Sweden t/a Natural Cycles. Available online at:

https://www.asa.org.uk/rulings/naturalcycles-nordic-ab-sweden-a17-393896.html

Bridges, Khiara (2011) Reproducing Race: An Ethnography of Pregnancy as a Site of Racialization. Berkeley: University of California Press.

Brown, Nik and Webster, Andrew (2004) New Medical Technologies and Society: Reordering Life. Cambridge: Polity Press.

Butler, Judith (1993) Bodies That Matter: On the Discursive Limits of "Sex". New York: Routledge, 2014.

Clarke, Adele (2000) Maverick reproductive scientists and the production of contraceptives, 1915-2000+. In: Saetnan, Oudshoorn and Kirejczyk (eds) Bodies of Technology: Women's Involvement with Reproductive Medicine. Columbus: Ohio State University Press.

Cowan, Ruth S. (1983) More Work for Mother: The Ironies of Household Technology from the Open Hearth to the Microwave. New York: Basic Books. 
Journal: Body \& Society

Author: Dr Josie Hamper

Davis, Dana-Ain (2009) The politics of reproduction: The troubling case of Nadya Suleman and assisted reproductive technology. Transforming Anthropology, 17(2): 105116.

Davis-Floyd, Robbie and Dumit, Joseph (eds) (1998) Cyborg Babies: From Techno-Sex to Techno-Tots. London: Routledge.

DeNora, Tia (1996) From physiology to feminism: Reconfiguring body, gender and expertise in natural fertility control. International Sociology, 11(3): 359-383.

Downe, Soo and Dykes, Fiona (2009) Counting time in pregnancy and labour. In: McCourt (ed) Childbirth, Midwifery and Concepts of Time. London: Berghahn Books.

Epstein, Charlotte (2016) Surveillance, privacy and the making of the modern subject: Habeas what kind of corpus. Body \& Society, 22(2): 28-57.

Evans, Bethan (2010) Anticipating fatness: Childhood, affect and the pre-emptive 'war on obesity'. Transactions of the Institute of British Geographers, 35(1): 21-38.

Evans, Geoffrey and Mellon, Jonathan (2016) Social Class: Identity, awareness and political attitudes: why are we still working class? NatCen Social Research: British Social Attitudes 33. Available online at: http://www.bsa.natcen.ac.uk/latest-report/britishsocial-attitudes-33/social-class.aspx. 
Journal: Body \& Society

Author: Dr Josie Hamper

Franklin, Sarah (1995) Postmodern procreation: A cultural account of assisted reproduction. In: Ginsburg and Rapp (eds) Conceiving the New World Order: The Global Politics of Reproduction. London: University of California Press.

Franklin, Sarah (1997) Embodied Progress: A Cultural Account of Assisted Conception. Oxon: Routledge.

Gillespie, Tarleton (2014) The relevance of algorithms. In: Gillespie, Boczkowski and Foot (eds) Media Technologies: Essays on Communication, Materiality, and Society. London: MIT Press.

Ginsburg, Faye and Rapp, Rayna (eds) (1995) Conceiving the New World Order: The Global Politics of Reproduction. London: University of California Press.

Han, Sallie (2013) Pregnancy as a literacy event. In: Han, Pregnancy in Practice: Expectation and Experience in the Contemporary US. Oxford: Berghahn Books.

Haraway, Donna (1988) Situated knowledges: The science question in feminism and the privilege of partial perspective. Feminist Studies, 14(3): 575-599.

Holloway, Sarah L. and Pimlott-Wilson, Helena (2014) "Any advice is welcome isn't it?": Neoliberal parenting education, local mothering cultures, and social class. Environment and Planning A: Economy and Space, 46(1): 94-111. 
Journal: Body \& Society

Author: Dr Josie Hamper

Kitchin, Rob and Dodge, Martin (2011) Code/Space: Software and Everyday Life. Cambridge: MIT Press.

Lee, Ellie (2014) Experts and parenting culture. In: Lee, Bristow, Faircloth and Macvarish (eds) Parenting Culture Studies. Hampshire: Palgrave Macmillan.

Lock, Margaret and Nguyen, Vinh-Kim (2010) Part I: Technologies and bodies in context. In: An Anthropology of Biomedicine. Chichester: Wiley-Blackwell.

Longhurst, Robyn (2008) Maternities: Gender, Bodies and Space. Oxon: Routledge.

Lupton, Deborah (2012) M-health and health promotion: The digital cyborg and surveillance society. Social Theory \& Health, 10(3): 229-244.

Lupton, Deborah (2013) The digitally engaged patient: Self-monitoring and self-care in the digital health era. Social Theory \& Health, 11(3): 256-270.

Lupton, Deborah (2014a) Quantified sex: A critical analysis of sexual and reproductive self-tracking using apps. Culture, Health \& Sexuality, 17(4): 440-453,

Lupton, Deborah (2014b) Apps as artefacts: Towards a critical perspective on mobile health and medical apps. Societies, 4(4): 606-622. 
Journal: Body \& Society

Author: Dr Josie Hamper

Lupton, Deborah (2015) 'Mastering Your Fertility': The Digitised Reproductive Citizen. In: McCosker, Vivienne and Johns (eds) Negotiating Digital Citizenship: Control, Contest and Culture. London: Rowman and Littlefield. Available online at:

$\underline{\text { https: } / / \text { ssrn.com/abstract }=2679402}$

Lupton, Deborah (2017) Introduction: Digitised health, medicine and risk. In: Lupton (ed) Digitised Health, Medicine and Risk. London: Routledge.

Marshall, Harriette and Woollett, Anne (2000) Fit to reproduce? The regulative role of pregnancy texts. Feminism \& Psychology, 10(3): 351-366.

Martin, Emily (1987) The Woman in the Body: A Cultural Analysis of Reproduction. Boston: Beacon Press.

Martin, Emily (1991) The egg and the sperm: How science has constructed a romance based on stereotypical male-female roles. Signs: Journal of Women in Culture and Society, 16(3): 485-501.

Martin, Lauren J. (2017) Pushing for the perfect time: Social and biological fertility. Women's Studies International Forum, 62: 91-98.

McRobbie, Angela (2015) Notes on the perfect: Competitive femininity in neoliberal times. Australian Feminist Studies, 30(83): 3-20. 
Journal: Body \& Society

Author: Dr Josie Hamper

Rettberg, Jill W. (2014) Privacy and surveillance. In: Rettberg, Seeing Ourselves Through Technology. Hampshire: Palgrave Macmillan.

Roberts, Celia (2007) Messengers of Sex: Hormones, Biomedicine and Feminism.

Cambridge: Cambridge University Press.

Rose, Nik and Novas, Carlos (2005) Biological citizenship. In: Ong and Collier (eds)

Global Assemblages: Technology, Politics and Ethics as Anthropological Problems. Oxford: Blackwell.

Rottenberg, Catherine (2014) The rise of neoliberal feminism. Cultural Studies, 28(3): 418-437.

Ruckenstein, Minna (2014) Visualized and interacted life: Personal analytics and engagements with data doubles. Societies, 4(1): 68-84.

Sanders, Rachel (2016) Self-tracking in the digital era: Biopower, patriarchy, and the new biometric body projects. Body \& Society, 23(1): 36-63.

Sawchuk, Kim (2000) Biotourism, Fantastic Voyage, and sublime inner space. In: Marchessault, Janine and Sawchuk, Kim (eds) Wild Science: Reading Feminism, Medicine and the Media. London: Routledge. 
Journal: Body \& Society

Author: Dr Josie Hamper

Simonds, Wendy (2002) Watching the clock: Keeping time during pregnancy, birth, and postpartum experiences. Social Science \& Medicine, 55: 559-570.

Smith, Gavin J. D. and Vonthethoff, Ben (2016) Health by numbers? Exploring the practice and experience of datafied health. Health Sociology Review, 26(1): 6-21.

Teather, Elizabeth K. (1999) Introduction: Geographies of personal discovery. In: Teather (ed) Embodied Geographies: Spaces, Bodies and Rites of Passage. London: Routledge.

Thompson, Charis (2005) Making Parents: The Ontological Choreography of Reproductive Technologies. Cambridge: MIT Press.

Tyler, Imogen (2008) "Chav mum chav scum". Feminist Media Studies, 8(1): 17-34.

van Dijck, José (2005) The Transparent Body: A Cultural Analysis of Medical Imaging. Seattle: University of Washington Press.

van Dijck, José (2009) Users like you? Theorizing agency in user-generated content. Media Culture Society, 31(1): 41-58.

Waggoner, Miranda (2015) Cultivating the maternal future: Public health and the prepregnant self. Signs: Journal of Women in Culture and Society, 40(4): 939-962. 
Journal: Body \& Society

Author: Dr Josie Hamper

Wilkinson, Joann, Roberts, Celia and Mort, Maggie (2015) Ovulation monitoring and

reproductive heterosex: Living the conceptive imperative? Culture, Health \& Sexuality,

17(4): 454-469.

\footnotetext{
1 The Natural Cycles app became a focal point for these critiques due to its extensive targeted social media advertising strategy, which emphasised the accuracy of the app's predictive algorithm as comparable to other contraceptive methods but did not specify that this was only the case for perfect-use (ASA, 2018).

2 People's sense of class identification in the UK is grounded in their occupation, education, socioeconomic and family background, and sociopolitical views (Evans and Mellon, 2016). Intersecting across these categories are particular class-specific family and parenting aspirations, cultures and stereotypes (Tyler, 2008; Holloway and Pimlott-Wilson, 2014) that impact on how women imagine their reproductive futures.

${ }^{3}$ Participants had various experiences of sharing, or deciding not to share, their fertility tracking and knowledge with male partners. See the work of Joann Wilkinson et al. (2015) for an analysis of how ovulation sensing technologies are involved in producing normative articulations and performances of 'reproductive heterosex'.

4 The participant is likely to be referring to school biology lessons here rather than the sex and relationship education that takes place from the age of 11 in the UK.
} 